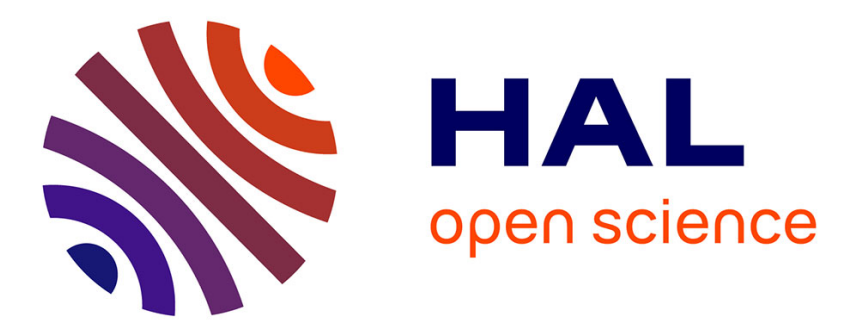

\title{
Some links between continuous and discrete Radon transform
}

\author{
Myriam Servières, N. Normand, P. Subirats, J.P. Guedon
}

\section{To cite this version:}

Myriam Servières, N. Normand, P. Subirats, J.P. Guedon. Some links between continuous and discrete Radon transform. Medical Imaging 2004, 2004, San Diego, United States. pp.1951-1960, 10.1117/12.533472 . hal-01500583

\section{HAL Id: hal-01500583 \\ https://hal.science/hal-01500583}

Submitted on 16 May 2017

HAL is a multi-disciplinary open access archive for the deposit and dissemination of scientific research documents, whether they are published or not. The documents may come from teaching and research institutions in France or abroad, or from public or private research centers.
L'archive ouverte pluridisciplinaire HAL, est destinée au dépôt et à la diffusion de documents scientifiques de niveau recherche, publiés ou non, émanant des établissements d'enseignement et de recherche français ou étrangers, des laboratoires publics ou privés. 


\title{
Some links between continuous and discrete Radon transform
}

\author{
Myriam Servières, Nicolas Normand, Peggy Subirats, and JeanPierre Guédon \\ IRCCyN - UMR CNRS 6597, Image \& Video Communication, \\ École polytechnique de l'Université de Nantes \\ La Chantrerie, BP 50609, 44306 Nantes Cedex 3
}

\begin{abstract}
The Filtered BackProjection is still questionable since many discrete versions have been derived from the continuous Radon formalism.

From a continuous point of view, a previous work has made a link between continuous and discrete FBP versions denoted as Spline 0-FBP model leading to a regularization of the infinite Ramp filter by the Fourier transform of a trapezoidal shape. However, projections have to be oversampled (compared to the pixel size) to retrieve the theoretical properties of Sobolev and Spline spaces. Here we obtain a novel version of the Spline 0 FBP algorithm with a complete continuous/discrete correspondence using a specific discrete Radon transform, the Mojette transform.

From a discrete point of view, the links toward the FBP algorithm are shaped with the morphological description and the extended use of discrete projection angles. The resulting equivalent FBP scheme uses a selected set of angles which covers all the possible discrete Katz's directions issued from the pixels of the (square) shape under reconstruction: this is implemented using the corresponding Farey's series. We present a new version of a discrete FBP method using a finite number of projections derived from discrete geometry considerations. This paper makes links between these two approaches.
\end{abstract}

Keywords: Tomographic Reconstruction, Discrete Tomography, FBP, Discrete Geometry, Mojette Transform

\section{INTRODUCTION}

Many discrete versions implementing the Filtered BackProjection have been derived from the continuous Radon formalism. ${ }^{?}$ But an infinite number of continuous projections is required to reconstruct a continuous $2 \mathrm{D}$ function belonging to an Euclidean space.? Several ways exist to discretize this problem. The Radon transform can be computed with direct methods (FBP algorithm ${ }^{?}$ or Fourier slice theorem ${ }^{?}$ ) or iterative methods (ART or OSEM $^{?}$ ) with possibly a priori information onto the object or the noise. On another side, discrete tomography uses a priori information on discrete geometry for the acquisition model and objects under reconstruction. The goal of this paper is to make some links between these two classical approaches.

In Section ??, from the continuous point of view, we will derive the proper way to sample the projections and the image domains in order to obtain a discrete FBP scheme. This scheme extends Guédon and Bizais work? by adapting the whole sampling scheme and therefore using the Mojette discrete Radon transform version. Section ?? shows the FBP Mojette result onto tests images and null-space example. Finally in section ??, from a pure discrete point of view, we present a new version of a discrete FBP method using a finite number of projections derived from discrete geometry considerations.

\section{FROM CONTINUOUS TO DISCRETE FBP}

\subsection{Continuous FBP}

In a classic point of view, FBP reconstruction scheme is processed onto projection given by uniform projection angle directions. The bin number on each projection is generally given by the image size. For an infinity of projections, FBP algorithm is summarized as:

$$
f(x, y)=R^{*} K R f(x, y)
$$

Phone: + 33240683 048, Fax: +33240683 232

E-mail: \{myriam.servieres, nicolas.normand, peggy.subirats, jean-pierre.guedon\}@polytech.univ-nantes.fr 
with the operators $R^{*}, K$ and $R$ defined as follows.

The Radon operator onto image $f(x, y)$ gives projections $p_{f}(t, \theta)$ with $t=-x \sin \theta+y \cos \theta$ :

$$
p_{f}(t, \theta)=\int_{-\infty}^{+\infty} \int_{-\infty}^{+\infty} f(x, y) \delta(t+x \sin \theta-y \cos \theta) d x d y
$$

The $K$ operator, representing the filtering part, is applied onto projections by convolution in the spacial domain or by multiplication in the Fourier domain. Each projection is convolved with the inverse Fourier transform of the infinite Ramp :

$$
\tilde{p}_{f}(t, \theta)=p_{f}(t, \theta)^{*} \mathcal{F}^{-1}(\pi|\nu|) .
$$

The filter is taken as rotationally invariant because no angular sampling is required at this step.

The backprojector operator $R^{*}$ is the dual of the Radon operator:

$$
R^{*} \tilde{p}_{f}(t, \theta)=R^{*} K p_{f}(t, \theta)
$$

Using an infinite number of projections and interpolations onto the projections and possibly oversampling, the $f(x, y)$ image is reconstructed.

\subsection{The FBP Mojette, a link between discrete and continuous spaces}

The Mojette FBP is based on the Mojette projector $M$ and its dual operator $M^{*}$.

\subsubsection{Mojette Projector}

Initially, the Mojette operator was defined from a discrete point of view ${ }^{?}$. The Mojette transform of a region gives projections only for direction $\tan \theta=\frac{q}{p}$ where $p$ and $q$ are integers (moreover, a single value of the angle class is kept by restricting $p$ and $q$ to be prime to each other and $q$ to be positive except for $p=1, q=0$ ).

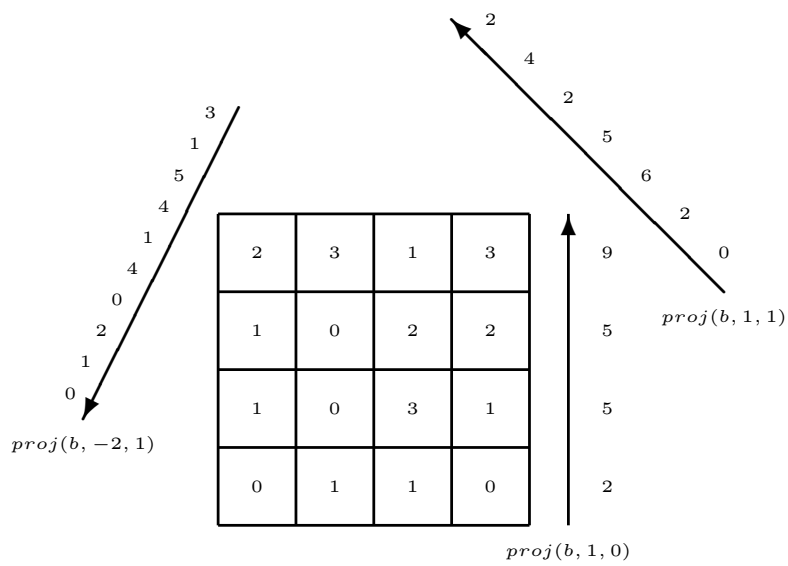

Figure 1. Mojette projections of a $4 \times 4$ image with projections directions $(p, q) \in\{(1,0),(1,1),(-2,1)\}$

It is defined as (Fig. ??):

$$
\operatorname{proj}(b, p, q)=\sum_{k=-\infty}^{+\infty} \sum_{l=-\infty}^{+\infty} f(k, l) \Delta(b+q k-p l)
$$

where $\Delta(b)=\left\{\begin{array}{l}0 \text { if } b \neq 0 \\ 1 \text { if } b=0\end{array}\right.$

where $b$ is called a bin. Using such an angle with the projection sampling leads to a regular lattice which projects 
all pixels centers onto projection bins. The projection size depends on the $(p, q)$ direction.

One of the main point in the Mojette definition, apart from the sampling, is the pixel model used to link continuous and discrete images. Choosing a pixel model gives a Mojette operator. For instance, the discrete Mojette definition (Eq.??) is equivalent to the properly discretized Radon transform with a Dirac pixel model.

Using a Dirac pixel model $\delta(x, y)=\delta(x) \delta(y)$, a continuous image $f(x, y)$ can be expressed as:

$$
f(x, y)=\sum_{k} \sum_{l} f(k, l) \delta(x-k) \delta(y-l) .
$$

Using this image definition in (Eq.??) with Katz angular sampling? $\tan \theta=\frac{q}{p}$ and the Mojette projection sampling $t=b / \sqrt{p^{2}+q^{2}}$ (inversely proportional to the length of vector $(p, q)$ ) gives again the Dirac Mojette operator defined in (Eq.??). Linearity and shift-invarianceness of the Radon transform are kept because both angular and projection samplings are adequately implemented.

Because of the Mojette sampling the linear system expressed in (Eq.??) is exactly invertible. From a rectangular image $Q \times P$, this matrix inversion allows to perform an inverse Mojette transform. A sufficient condition to invertibility is to have a minimum number of $I$ projections in the set $S=\left\{\left(p_{i}, q_{i}\right), i \in \mathbb{N}\right\}$ of all projections.? For the rectangular shape, the Katz criterion allows reconstruction if $P \leq \sum_{i=1}^{I}\left|p_{i}\right|$ or $Q \leq \sum_{i=1}^{I}\left|q_{i}\right|$ where $I$ is the number of projections.

In other words, the reconstruction does not depend directly on the number of bins but is first related to the number of projections. These reconstruction conditions have been generalized for any convex shape using a simple morphological tool: a two pixel structuring element that depicts a discrete direction.?

In this context, the inverse Mojette operator is also defined in a specific way. Instead of a matrix inversion, the operator only proceeds by finding at each iteration a discrete corner of the shape under reconstruction which is the only projected pixel onto a bin: the bin value is exactly backprojected and all projections are updated. The algorithm complexity of both the direct and inverse Mojette transform is $O(I N)$ where $N$ is the number of pixels. This inverse algorithm could only be used to reconstruct perfect data. If the data are noisy the reconstruction process become highly unstable? and the ill posed nature of the inverse Radon transform appears.

\subsubsection{Mojette Backprojector}

The Mojette backprojector is defined ${ }^{?}$ as the dual of the Mojette projector. For a Dirac pixel model, (Eq.??) is written as:

$$
\tilde{f}(k, l)=\sum_{p, q} \sum_{b} \operatorname{proj}(b, p, q) \Delta(b+q k-p l) .
$$

That means for one projection $(p, q): f_{p, q}(k, l)=M_{\delta}^{*} \operatorname{proj}(b, p, q)$ The Mojette dual operator $M^{*}$ corresponds to an exact discrete backprojector operator as show in (Fig. ??). Then, using a Mojette angle sampling allows to back-project onto pixel centers without any interpolation.

\subsection{Continuous-Discrete Equivalence}

In this paragraph, we first focus on the Spline 0 implementation of the General Sampling Theorem? ${ }^{\text {of Aldroubi }}$ and Unser. The corresponding Mojette operator denoted $M_{0}$ is derived. Then the same treatment is applied onto the filter. 


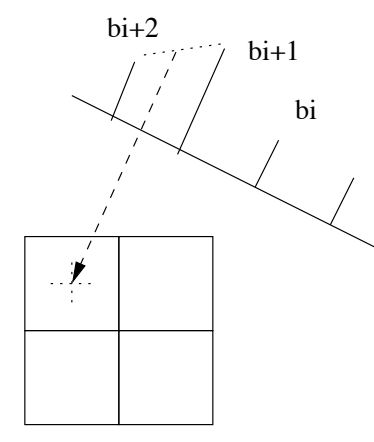

(a)

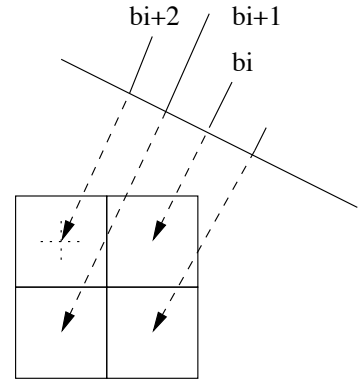

(b)

Figure 2. (a) Interpolation onto the projection in order to backproject the right value at the corresponding center of the pixel, (b) Exact Mojette backprojector.

\subsubsection{Spline 0 Pixel Model}

The Splines spaces (since they represent a reproducing kernel of Sobolev spaces) will be the choice to project the discrete Radon solutions issued from our derived schemes. Because of the recurrent relationships between Spline orders, only Splines of order 0 are considered here without loss of generality ${ }^{?}$. In other words, the formulation for a continuous function $f(x, y)$ from samples $f(k, l)$ is given following Aldroubi-Unser as ${ }^{\text {: }}$ :

$$
f_{0}(x, y)=\sum_{k=-\infty}^{+\infty} \sum_{l=-\infty}^{+\infty} f(k, l) \beta_{0}(x-k) \beta_{0}(y-l)
$$

where function $\beta_{0}$ is the step function of unitary length: $\beta_{0}(t)=\left\{\begin{array}{l}1 \text { if }|t|<\frac{1}{2} \\ 1 \text { if }|t|=\frac{1}{2} \\ 0 \text { elsewhere }\end{array}\right.$ Using (Eq.??) in (Eq.??) another version of the Mojette operator is obtained and denoted the Spline 0 Mojette $\left(M_{0}\right)$ :

$$
M_{0} f(k, l)=\operatorname{proj}_{0}(b, p, q)=\sum_{k=-\infty}^{+\infty} \sum_{l=-\infty}^{+\infty} f(k, l) \text { kernel }_{0}(k, l, b, p, q),
$$

with kernel $_{0}(k, l, b, p, q)=\int_{-\infty}^{+\infty} \int_{-\infty}^{+\infty} \beta_{0}(x-k) \beta_{0}(y-l) \delta(b+q x-p y) d x d y$.

Here kernel $_{0}$ corresponds to a discrete trapezoidal filter expressed as the convolution of two series of unitary values of respective lengths $p$ and $q$. It allows a link between Dirac Mojette transform and Spline 0 Mojette transform?

$$
\operatorname{proj}_{0}(b, p, q)=\operatorname{proj}_{\delta}(b, p, q) * \operatorname{kernel}_{0}(b, p, q)
$$

kernel $_{0}(b, p, q)$ is in fact the projection of the Spline 0 pixel model in the $(p, q)$ direction and it is a discrete trapezoid:

$$
\text { kernel }_{0}(b, p, q)=\left\{\begin{array}{l}
1 \text { if } p=0 \text { or } q=0 \\
\frac{1}{p q} \underbrace{(1 . .1)}_{p} * \underbrace{(1 . .1)}_{q} \text { if } p \text { and } q \text { are odd } \\
\frac{1}{2 p q} *(11) * \underbrace{(1 . .1)}_{p} * \underbrace{(1 . .1)}_{q} \text { if } p \text { or } q \text { is even. }
\end{array}\right.
$$


We also define the Spline $n$ Mojette operator $M_{n}$ using:

$$
M_{n} f(k, l)=\operatorname{proj}_{n}(b, p, q)=\sum_{k=-\infty}^{+\infty} \sum_{l=-\infty}^{+\infty} f(k, l) \operatorname{kernel}_{n}(k, l, b, p, q)
$$

with

$$
\operatorname{kernel}_{n}(k, l, b, p, q)=\int_{-\infty}^{+\infty} \int_{-\infty}^{+\infty} \beta_{n}(x-k) \beta_{n}(y-l) \delta(b+q x-p y) d x d y .
$$

\subsection{2. $k_{0}$ Filter}

To be consistent with the medical reconstruction problem which deals with continuous integration line rather than discrete ones as the Mojette transform does, the use of a Spline 0 pixel model and a discrete set of angles obtained from the acquisition model is mandatory. The Spline 0 Mojette operator that corresponds to model the bin value by the entire continuous line summation (not the discrete line as the Dirac Mojette operator) will

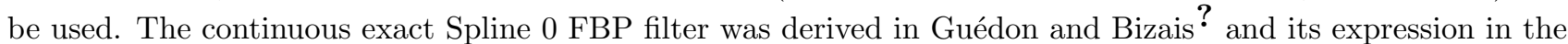
Fourier domain is given by:

$$
K_{0}(\nu, \theta)=\pi|\nu| \operatorname{sinc}(\nu \cos \theta) \operatorname{sinc}(\nu \sin \theta),
$$

where the apodisation function corresponds to the projection of the filter onto the Spline space of order 0. The pixel size is unitary.

The inverse fourier transform of (Eq.??) was derived as

$$
k_{0}(t, \theta)=\frac{1}{\pi \sin (2 \theta)} \ln \left|\frac{t^{2}-\left(\frac{1+\sin (2 \theta)}{4}\right)}{t^{2}-\left(\frac{1-\sin (2 \theta)}{4}\right)}\right|,
$$

for $t \neq 0$ and $\left.\theta \in] 0, \frac{\pi}{4}\right]$ and $k_{0}(t, 0)=\frac{-1}{\pi} \frac{2}{4 t^{2}-1}$.

Discretizing with Mojette angles $\left(\tan \theta=\frac{q}{p}\right)$ with the projection sampling $t=\frac{b}{p^{2}+q^{2}}$ leads to:

$$
k_{0}(b, p, q)=\frac{p^{2}+q^{2}}{2 \pi p q} \ln \left|\frac{b^{2}-\left(\frac{p+q}{2}\right)^{2}}{b^{2}-\left(\frac{p-q}{2}\right)^{2}}\right|,
$$

for $b \neq 0$ and $(p, q) \neq(1,0)$ and $k_{0}(b, 1,0)=\frac{-1}{\pi} \frac{2}{4 b^{2}-1}$.

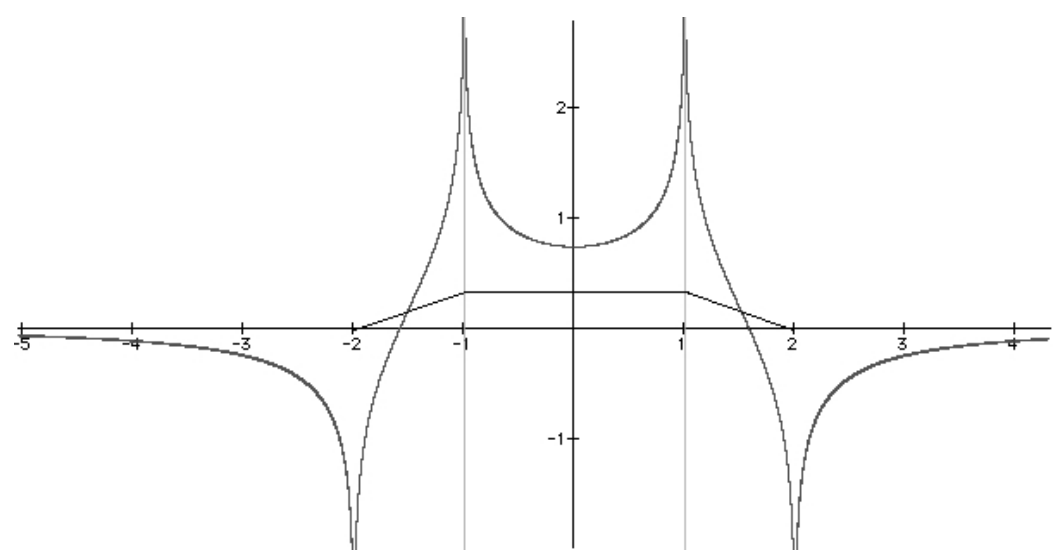

Figure 3. Trapezoidal shape projection onto the $(1,3)$ direction and its corresponding $k_{0}$ filter. 
This filter can not be implemented in a straightforward manner in the spatial domain. It exhibits discontinuities at the points where the projection of the pixel (described by a trapezoidal shape) is continuous but not differentiable. The Ramp filter acting as a derivative operator, the values at these points have to be computed using a Dirichlet condition. Implementing the FBP Mojette operator uses the Mojette projector, the $k_{0}$ filter and the Mojette backprojector. This FBP Mojette scheme is exact for the filter when an infinite number of angles is used and exact for the backprojection of these filtered angles.

\subsection{Summary}

Fig.?? explicits somes links between the previous equations.

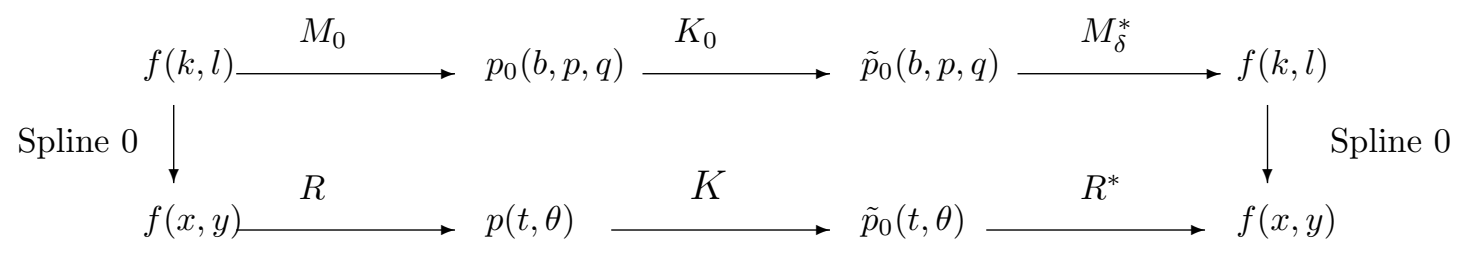

Figure 4. Equivalence between continuous and discrete scheme

The $K_{0}$ Fourier expression (Eq.??) is divided in two parts: a perfect Ramp filter and a trapezoidal shape. This trapeze is the projection of the Spline 0 pixel Model. (Eq.??) represents the use of the corresponding Riesz basis (constructed by tensorial product of $\beta_{0}$ ) to get the continuous-discrete equivalence of the original image $f(x, y)$. Applying the FBP Mojette scheme, the $f(k, l)$ is projected using the Spline 0 Mojette projection (Eq.??). The projections are convolved with the $k_{0}$ filter, i.e. with the perfect Ramp filter then with a trapezoid. The last stage is the Dirac Mojette backprojection (Eq.??). With (Eq.??) these stages are equivalent to a Spline 0 Mojette projection, a filtering with the Ramp filter and a Spline 0 Mojette backprojection.

This scheme ensures a continuous/discrete equivalence (in the Aldroubi and Unser sense) at each stage (Fig. ??). (Eq.??) and (Eq.??) define the Mojette operator for the Dirac and Spline 0 pixel intensity distribution model respectively. These links constitute the natural way of implementing an exact discrete FBP using an infinite number of projections. The resulting scheme is composed of a discrete projection operator using the Mojette sampling, a discrete Spline 0 Ramp filter with the same sampling, and a discrete exact backprojection operator $^{?}: M_{\delta}^{*} K_{0} M_{0}=I d_{0}$.

\section{FBP MOJETTE RESULTS AND EXTENSIONS}

\subsection{FBP Reconstruction}

The first test image for the FBP Mojette reconstruction is a $128 \times 128$ Dirac image. We use the same method as in ${ }^{?}$ computing 128 gracefully discrete angles with Farey's ${ }^{?}$ series. The Farey series of order $N\left(F_{N}\right)$ is the set of all fractions in lowest terms between 0 and 1 whose denominators do not exceed $N$, arranged in order of magnitude. For example, $F_{4}$ is composed of $\left\{\frac{0}{1}, \frac{1}{4}, \frac{1}{3}, \frac{1}{2}, \frac{2}{3}, \frac{3}{4}, \frac{1}{1}\right\}$. The FBP Mojette scheme was applied on the Dirac image with various number of projections (64, 128 and 256). It was also applied with Dirac and Spline 0 pixel model. The reconstructed images (Fig.??) are normalized and the line containing the Dirac is extracted.

A comparison is made between the FBP Mojette scheme with a Dirac and a Spline 0 Mojette projector, a $k_{0}$ filter and a Dirac Mojette backprojector. We observe the reconstruction differences onto a Dirac image with one or two trapezoids convolved onto the projections. With the $M_{\delta}^{*} K_{0} M_{0}$ the projections stay in the Sobolev space due to the Spline 0 pixel model. With the $M_{\delta}^{*} K_{0} M_{\delta}$ there is only one trapezoid in the filter and the resulting filtered projections are not in the Sobolev space, i.e. the continuous-discrete equivalence is not ensured.

For all three cases, the Dirac image obtained with the $M_{\delta}^{*} K_{0} M_{0}$ scheme is better reconstructed and smoother than the one obtained by a $M_{\delta}^{*} K_{0} M_{0}$ scheme. In fact, with the Mojette Spline 0 projector, the projections are smoothed one more time with a trapezoidal shape (Fig.??.b, Fig.??.d and Fig.??.f). With the Dirac Mojette 
projector, there are some irregularities in the reconstructed image around the Dirac (Fig.??.a, Fig.??.c and Fig.??.e). Fortunately, the reconstructed images are better when the number of projections is increasing.

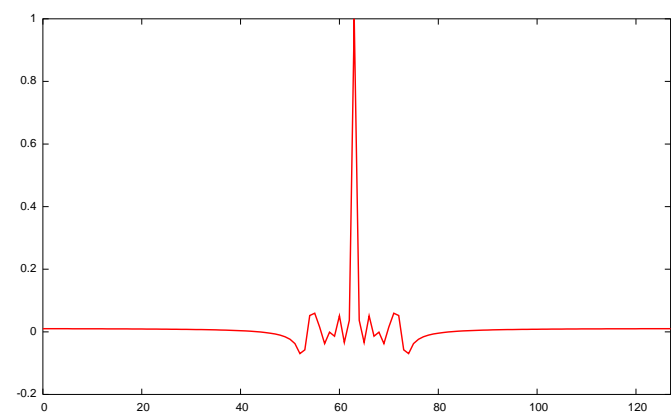

(a)

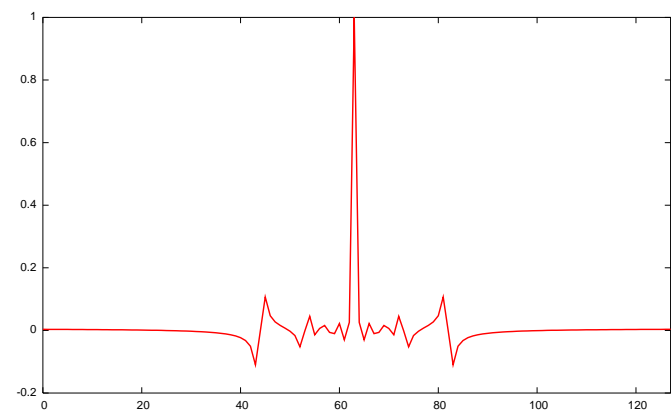

(c)

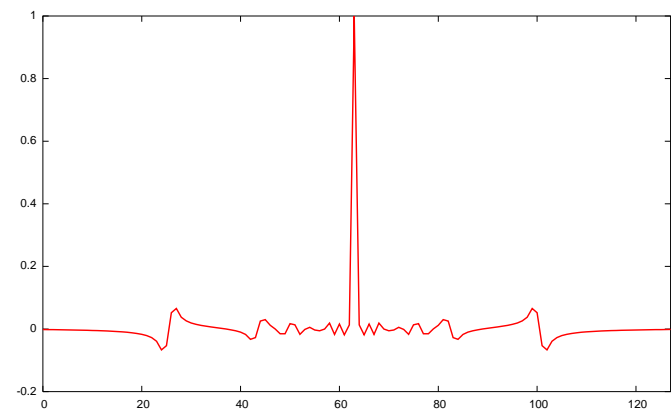

(e)

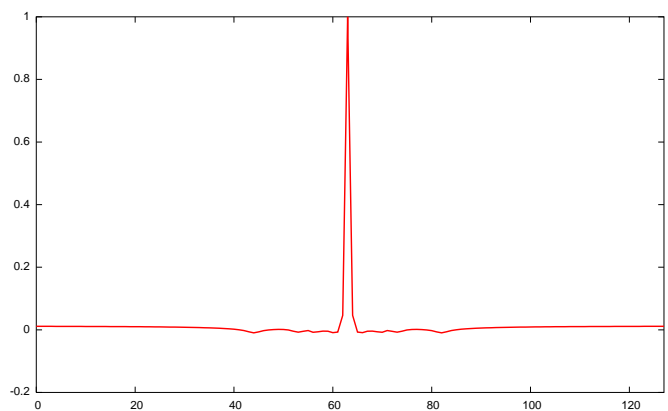

(b)

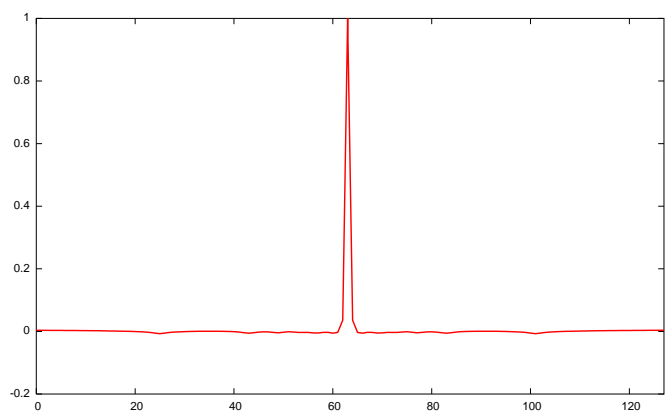

(d)

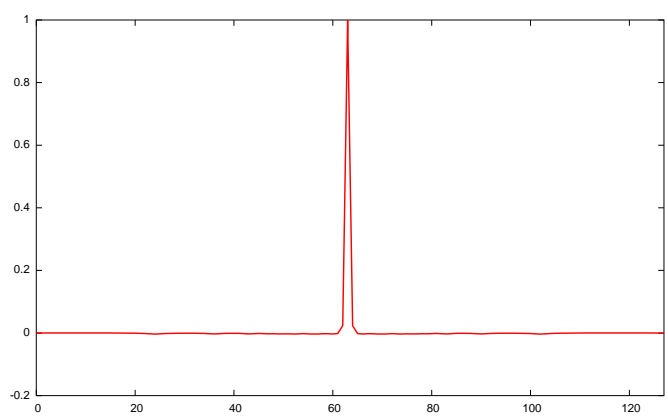

(f)

Figure 5. Slice of the Dirac reconstructed image with FBP Mojette. (a) Reconstructed image with 64 projections and a $M_{\delta}^{*} K_{0} M_{\delta}$ FBP Mojette scheme. (b) Reconstructed image with 64 projections and a $M_{\delta}^{*} K_{0} M_{0}$ FBP Mojette scheme. (c) Reconstructed image with 128 projections and a $M_{\delta}^{*} K_{0} M_{\delta}$ FBP Mojette scheme. (d) Reconstructed image with 128 projections and a $M_{\delta}^{*} K_{0} M_{0}$ FBP Mojette scheme. (e) Reconstructed image with 256 projections and a $M_{\delta}^{*} K_{0} M_{\delta}$ FBP Mojette scheme. (f) Reconstructed image with 256 projections and a $M_{\delta}^{*} K_{0} M_{0}$ FBP Mojette scheme.

\subsection{Null Space Analysis}

To obtain some vectors of the null space, we compute FBP Mojette algorithm onto two images (digital phantoms): first the Dirac image and second, a square $128 \times 128$ image with $17 \times 17$ centered pixels with a Dirichlet condition with the value 1 in the centered $15 \times 15$ square, $\frac{1}{2}$ in the boundaries and $\frac{1}{4}$ in the corners. We will now study 
the null space image of the FBP Mojette scheme $\left(M_{\delta}^{*} K_{0} M_{0}\right)$ by computing the difference image between the original and reconstructed images. Fig.?? summarizes the Dirac result whereas Fig.?? corresponds to the square analysis. Both phantoms were tested with 64, 128 and 256 projections. As shown on the difference images, as soon as the number of projections equals the size of the image, the degradations are both small and centered around the signal. The interest of the second phantom is to explicitely show edges degradations which is obvious there whereas difficult to interpret on the Dirac phantom. Fig.?? shows the reconstructed slice of the square phantom. The higher the projections number, the better the sharpness of the edges.

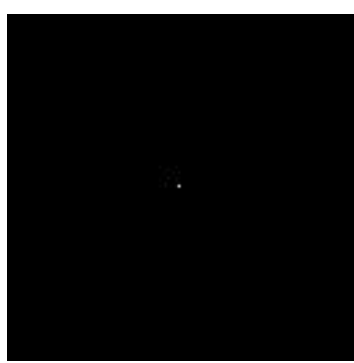

(a)

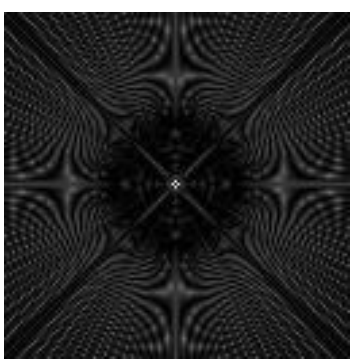

(b)

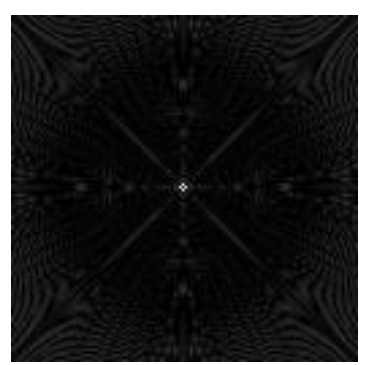

(c)

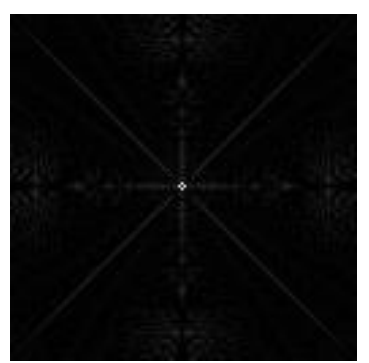

(d)

Figure 6. Dirac image reconstruction image with a $M_{\delta}^{*} K_{0} M_{0}$ FBP Mojette scheme. (a) Original image. (b) Normalized difference between original and reconstructed image for 64 projections (normalization factor $\frac{1}{0.046}$ ). (c) Normalized difference between original and reconstructed image for 128 projections (normalization factor $\frac{1}{0.036}$ ). (d) Normalized difference between original and reconstructed image for 256 projections (normalization factor $\frac{1}{0.023}$ )

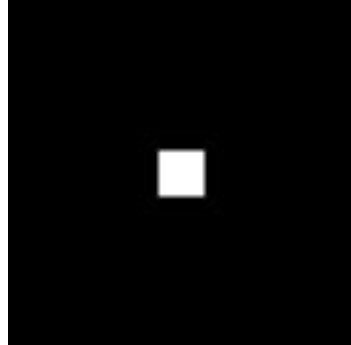

(a)

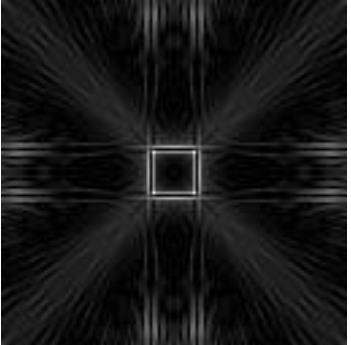

(b)

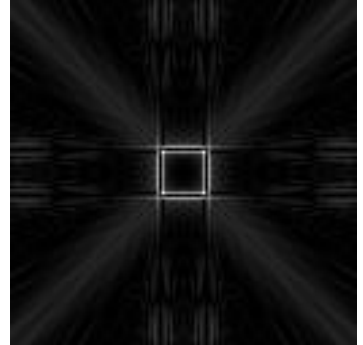

(c)

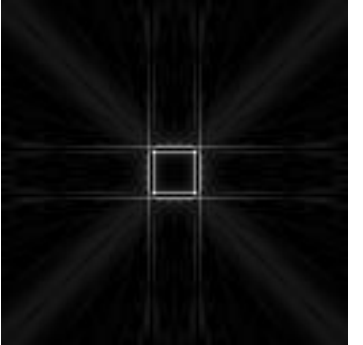

(d)

Figure 7. Square image reconstruction image with a $M_{\delta}^{*} K_{0} M_{0}$ FBP Mojette scheme. (a) Original image. (b) Normalized difference between original and reconstructed image (normalization factor $\frac{1}{0.098}$ ). (c) Normalized difference between original and reconstructed image (normalization factor $\frac{1}{0.070}$ ). (d) Normalized difference between original and reconstructed image (normalization factor $\frac{1}{0.050}$ ).

\subsection{Mojette Reconstruction}

A number of projections satisfying the Katz criteria is sufficient with the inverse Mojette operator (section ??) $M^{-1}$ to obtain an exact reconstruction. However, this reconstruction only operates onto perfect data. When noisy data are used, the reconstruction process diverges and the ill-posed nature of the Radon transform appears. In our case, we reconstruct the square image using 48 projections and satisfying Katz criteria. For the FBP like case the noise is filtered out (the background is almost zero, the square grey levels are almost flat) whereas the inverse Mojette transform produces an image with 0.5 mean value almost everywhere and some black and white mixed values at the square location. These experiments show that the characteristic vectors of the null space of the Mojette linear operator can change from an empty space (no noise and enough projections) to very complicated shapes when noise enters the game. A theoretical solution to this kind of problems can be found by deriving the exact discrete filter corresponding to our implementation of $M^{*} M$. 


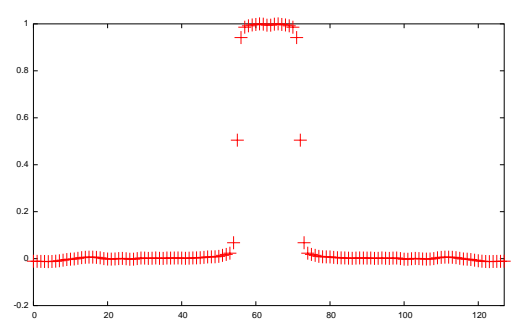

(a)

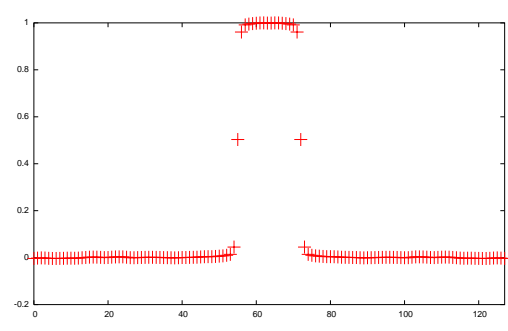

(b)

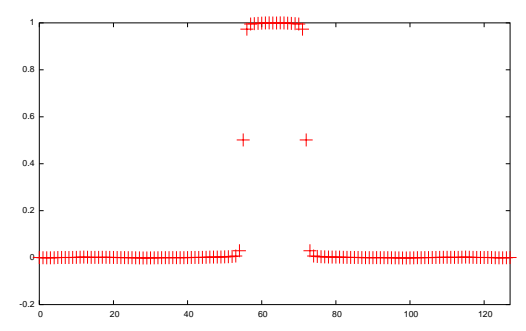

(c)

Figure 8. Slice in the middle of the square of the normalized reconstructed square image with various number of projections. (a) 64 projections. (b) 128 projections. (c) 256 projections.

\section{PURE DISCRETE FBP APPROACH}

When a discrete set of pixels is described from a finite number of projections, a priori geometric features information is mandatory. In our case, the links toward the FBP algorithm are constituted by the morphological description and the extended use of discrete projection angles. The mathematical morphology has been used through the two-pixel structuring element object that is strongly related to the direction of a projection ${ }^{?}$ and allows for deriving the geometrical conditions under which a set of connected pixels can be reconstructed from a given set of Katz's projections. These sets of projections generalize the usual set of two (0 and 90$)$ or four projections found in the subsequent literature. The resulting equivalent FBP scheme uses a selected set of angles which covers all the possible discrete Katz's directions issued from the pixels of the (square) shape under reconstruction: this is implemented using the corresponding Farey's series. For instance a $128 \times 128$ image will need $\mathrm{I}=20088$ directions (i.e. $\operatorname{Card}($ Farey $(128))$ to cover $[0, \pi[$.

From the Dirac Mojette projection and backprojection definitions we derive the value of the pixel $(k, l)$ in the image obtained by backprojecting the projection $i$ :

$$
\tilde{f}_{i}(k, l)=\sum_{k^{\prime}} \sum_{l^{\prime}} f\left(k^{\prime}, l^{\prime}\right) \Delta\left(q_{i} k-p_{i} l-q_{i} k^{\prime}+p_{i} l^{\prime}\right) .
$$

This can be expressed as the sum of pixels aligned with $(k, l)$. Considering a set of projections $\left\{\left(p_{i}, q_{i}\right)\right\}$, the value of pixel $(k, l)$ becomes:

$$
\tilde{f}(k, l)=I f(k, l)+\sum_{k^{\prime} \neq k} \sum_{l^{\prime} \neq l} f\left(k^{\prime}, l^{\prime}\right) \Delta\left(q_{i} k-p_{i} l-q_{i} k^{\prime}+p_{i} l^{\prime}\right)
$$

Using all the projections corresponding to all the projections needed to go from one pixel to one other in the image. Every pixel $\left(k^{\prime}, l^{\prime}\right) \neq(k, l)$ is backprojected once in $(k, l)$. This leads to:

$$
\tilde{f}(k, l)=(I-1) f(k, l)+\sum_{i} \sum_{j} f(i, j)
$$

Since the sum of pixel values is equal to the sum of each projection bin values, the exact reconstruction (see Fig.??) is obtained by:

$$
f(k, l)=\frac{1}{I-1}\left[\tilde{f}(k, l)-\frac{1}{I} \sum_{i} \sum_{b} \operatorname{proj}\left(b, p_{i}, q_{i}\right)\right]
$$

Practically, a finite set of Farey angles is used. The order of the Farey series is taken equal to the image side. This a simple relationship acting as a filtering step that relies the original image to this set of projections via the same implementation of the exact backprojection. In other words, this scheme implements a filtering and a backprojection operators and gives back the original compact domain when the projector used is the dual of the backprojector, i.e. $M^{*} K_{F} M=I d$, where $K_{F}$ is the discrete filter indexed by the Farey's series. 


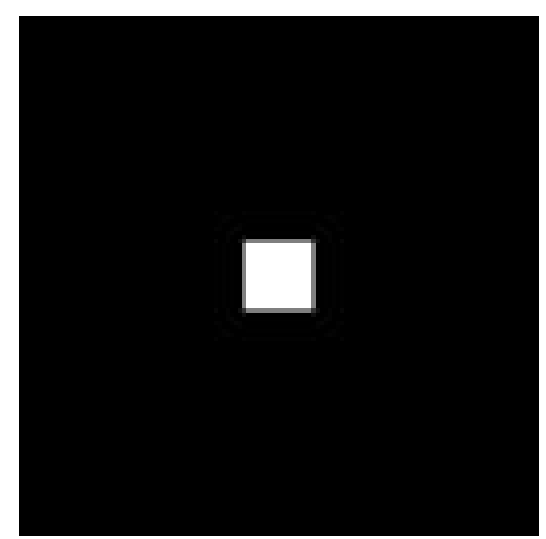

Figure 9. Exact reconstructed $128 \times 128$ square image with the pure discrete FBP approach (20088 projections).

\section{DISCUSSION}

Revisiting the angle sampling for the discrete tomographic problem gives us two novel algorithms based on the classical FBP reconstruction algorithm.

The first algorithm introduces the Spline 0 representation that can be easily extended to the whole Spline class. The Mojette operator $M_{\delta}$ and its dual $M_{\delta}^{*}$ act as the way to properly go onto the projection space and to come back in the image domain without loosing information. The resulting filter definition can be derived except at specific locations (points where the trapezoid is not differentiable) that can be approximated. Retrieving these singularities is good news: there were masqued in Guédon and Bizais ${ }^{?}$ by the conventional projection and angular sampling. The use of higher Spline degree will allow to obtain an exact scheme. However, the Aldroubi-Unser ${ }^{?}$ sampling theorem induces a postfilter (reduces to identity for Spline 0 ) after the reconstruction stage.

The whole discrete algorithm extends a step further this analysis. It allows for a perfect reconstruction with a finite (even if high) number of projections computed from the Farey's series of the image size. In other words, the filter is now defined from discrete constraints instead of coming from the sampling of the continuous Ramp filter. We thereby obtain the completely discrete FBP equation $M^{*} K M=I d$.

Because of its implementation it is quite difficult to obtain enough projections to use the latter algorithm. However the links between the two versions are strong (same kind of angles, same sampling onto the projections and image). This contucts us to compare the two filters and to use the latter algorithm within the context of a restricted number of projections (null spaces of both operators are constructed from the same eigenvectors deduced from $\left.M^{*} M\right)$.

The final goal is to interpret these relationships between the two operators in the sense that allows for optimizing the filter where only a small number of projections are available.

\section{CONCLUSION}

Two novel FBP like algorithms were presented and there links discussed. The differences in their final aspect give insight for the explicit null space decomposition when the number of projections will be low and for using the compactness constraint issued from the discrete side into the Spline 0 FBP method for reconstructing a region of interest. In order to go further in these new operators analysis, the noise generation will be now performed associated with the device acquisition accordance. This will imply to compute Mojette bin values from actual noisy data accumulated from a finite number of projections which are not distributed in a beautiful Farey's geometry. 